\title{
Câncer de pulmão: reabilitação
}

Autoria: Associação Brasileira de Medicina Física e Reabilitação

Elaboração Final: 21 de junho de 2013

Participantes: Rebeca Boltes Cecatto, Elisangela Marinho Pinto Almeida, Maíra Saul, Christina May Moran de Brito, Rodrigo Guimarães Andrade, Marta Imamura, Chennyfer Dobbins Paes da Rosa, Wanderley

Marques Bernardo, Linamara Rizzo Battistella

\section{DESCRIÇÃO DO MÉTODO DE COLETA DE EVIDÊNCIA}

Foram revisados artigos nas bases de dados do MedLine (PubMed) e outras fontes de pesquisa, sem limite de tempo. A estratégia de busca utilizada baseou-se em perguntas estruturadas na forma P.I.C.O. (das iniciais "Paciente", "Intervenção", "Controle", "Outcome").

Foram utilizados como descritores (MeSH terms):

PERGUNTA 1: Lung Neoplasms AND (Exercise Therapy OR Exercise OR Exercise Movement Techniques OR Resistance Training OR Muscle Stretching Exercises OR Breathing Exercises);

PERGUNTA 2: Lung Neoplasms AND Exercise OR Exercise Therapy AND Preoperative AND Pulmonary Complications;

PERGUNTA 3: Lung Neoplasms AND (Physical Therapy Modalities OR Exercise OR Exercise Therapy) AND (Dyspnea OR Breathing Disorders);

PERGUNTA 4: Lung Neoplasms AND (Physical Therapy Modalities OR (Exercise OR Exercise Therapy) AND Postoperative Period AND (Fatigue OR Cancer-Related Fatigue);

PERGUNTA 5: (Cognitive Therapy OR Psychoeducational Intervention) AND (Lung Neoplasms) AND (Quality of Life);

PERGUNTA 6: (Lung Neoplasms OR Lung Cancer) AND (Exercise OR Physical Fitness OR Exercise Therapy OR Physical Fitness OR Physical Activity) AND (Exertion OR Exercise Tolerance)

PERGUNTA 7: (Creatine Supplementation AND Neoplasm) OR (Creatine Supplementation AND Cancer) OR (HMB Supplementation AND Neoplasm) OR (HMB Supplementation AND Cancer)

PERGUNTA 8: ("Paullinia" OR Paullinia Cupana OR Guarana) AND (“Neoplasms"[Mesh] OR Cancer OR Fatigue)
Estes descritores foram usados para cruzamentos de acordo com o tema proposto em cada tópico das perguntas P.I.C.O. Após análise desse material, foram selecionados os artigos relativos às perguntas que originaram as evidências que fundamentaram a presente diretriz.

\section{GRAU DE RECOMENDAÇÃO E FORÇA DE EVIDÊNCIA:}

A: Estudos experimentais ou observacionais de melhor consistência.

B: Estudos experimentais ou observacionais de menor consistência.

C: Relatos de casos (estudos não controlados)

D: Opinião desprovida de avaliação crítica, baseada em consensos, estudo fisiológicos ou modelos animais.

\section{OBJETIVOS:}

Oferecer informações sobre o tratamento de reabilitação de pacientes com câncer de pulmão.

\section{PROCEDIMENTOS:}

Intervenções terapêuticas de reabilitação para as principais manifestações clínicas que comprometem a qualidade de vida, funcionalidade e atividades da vida cotidiana dos pacientes com neoplasia de pulmão, no âmbito biopsicossocial.

\section{CONFLITO DE INTERESSE:}

Nenhum conflito de interesse declarado.

\section{INTRODUÇÃO}

O câncer de pulmão é um dos tumores malignos mais frequentes no Brasil e no mundo. ${ }^{1,2}$ Em 2008, foi o câncer mais diagnosticado nos Estados Unidos e também a principal causa de morte oncológica em homens. ${ }^{1,2}$

No momento do diagnóstico, mais de $50 \%$ dos pacientes com câncer de pulmão já têm doença metastática. ${ }^{3}$

Quanto às manifestações clínicas, tosse, dispneia, hemoptise, dor torácica e rouquidão são sintomas frequentes. Tosse geralmente está

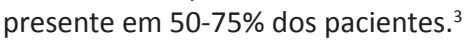

Dispneia também é um sintoma comum já no momento do diagnóstico, ocorrendo em aproximadamente $25 \%$ dos casos. ${ }^{3}$ Ela pode ser 
decorrente de: obstrução das vias aéreas extrínsecas ou intraluminal, pneumonite obstrutiva ou atelectasia, disseminação do tumor, linfangite, êmbolos do tumor, pneumotórax, derrame pleural e derrame pericárdico com tamponamento.

Essas e outras manifestações clínicas podem comprometer a funcionalidade e qualidade de vida, além de comumente estarem associados à fadiga nessa população.

Portanto, assim como em outros tipos de câncer e doenças crônicas, existe uma preocupação crescente em busca de opções terapêuticas que reduzam a fadiga, melhorem os sintomas clínicos, físicos e psíquicos e, com isso, melhorem a qualidade de vida.

Sendo assim, as intervenções de reabilitação, com destaque para o exercício terapêutico supervisionado, constituem potenciais alicerces para o tratamento adjuvante desta população. O Programa de Exercício Supervisionado constitui uma intervenção terapêutica recomendada para muitos pacientes com câncer, entre eles, o câncer de pulmão, o que vem impulsionando os estudos científicos na área e ampliando o conhecimento sobre seu efeito nas diferentes manifestações clínicas.

O objetivo desta diretriz é avaliar a eficácia das estratégias de reabilitação para as condições clínicas mais comuns e de maior impacto na evolução clínica destes pacientes.

\section{SESSÃO TERAPÊUTICA}

\section{QUAIS SÃo OS EFEITOS do EXERCícIO Físico NOS PACIENTES COM CÂNCER DE PULMÃo?}

O treino supervisionado de fortalecimento e mobilidade, duas vezes ao dia, nos cinco primeiros dias do pós-operatório de cirurgia para resseção de neoplasia de pulmão, seguido de exercícios domiciliares, por um período de 12 semanas, realizados diariamente e baseados nas orientações feitas por um fisioterapeuta durante a internação (constituído de caminhada no limite do paciente, marcha estacionária e exercícios em bicicleta horizontal, com a intensidade entre $60 \%$ e $80 \%$ da frequência cardíaca máxima, por 5-10 minutos, associado a treino de fortalecimento de membros inferiores com carga inicial de 0,9 $\mathrm{kg}$ até $1,8 \mathrm{~kg})$, melhora a força do quadríceps $(p=0,04)$ nos primeiros cinco dias após a cirurgia, mas não reduz tempo de internação nem melhora a qualidade de vida $(p>0,05)$ nem a tolerância ao exercício $(p=0,89)^{4}(\mathbf{A})$.

Exercícios domiciliares, realizados diariamente no período préoperatório, não têm impacto positivo na percepção de saúde do paciente, mas melhora a capacidade de participar das mudanças e, consequentemente, pode ter efeito positivo na percepção do bemestar em pacientes com câncer de pulmão ${ }^{5}(\mathbf{A})$.

Exercícios respiratórios associados ao treino aeróbico por 30 minutos, com $50 \%$ da frequência cardíaca máxima, realizados diariamente, cinco dias por semana durante quatro semanas, em pacientes com doença pulmonar obstrutiva crônica, candidatos à ressecção de pulmão por câncer não pequenas células, melhoram a capacidade ao exercício $(p<0,001)^{6}$ (B).

Exercícios respiratórios associados à bicicleta estacionária, exercícios de membros superiores e inferiores de baixa a moderada intensidade e caminhada, supervisionados, com duração de 90 minutos, diariamente, por um período de sete semanas, aumenta a função física, embora não melhore a capacidade pulmonar e qualidade de vida em pacientes com doença pulmonar obstrutiva crônica e câncer de pulmão inoperável ${ }^{7}$ (B).

Pacientes com diversos tipos de câncer melhoram a fadiga após programa com treino aeróbico, associado a exercícios resistidos de tronco, membros superiores e inferiores (com carga de treinamento entre $30-100 \%$ de uma repetição máxima), numa frequência mínima de duas vezes por semana, no período pós-tratamento com radioterapia ou quimioterapia ${ }^{8,9}(\mathbf{A})$ e também pacientes com câncer de próstata, durante a radioterapia, têm benefícios na qualidade de vida com um programa de exercícios aeróbicos supervisionado associado ao treino resistido ${ }^{10}(\mathrm{~A})$.

O exercício aeróbico supervisionado com intensidade de $80 \%$ da frequência cardíaca máxima, realizado diariamente, por três semanas, reduz os sintomas de fadiga em pacientes com diversos diagnósticos de câncer (pulmão, estômago, cólon e reto) após o tratamento cirúrgico ${ }^{11}$ (B). O treino aeróbico também tem efeitos benéficos na qualidade de vida em pacientes sobreviventes de câncer de $\operatorname{mama}^{12}$ (A).

Um programa de exercícios resistidos melhora o bem-estar em pacientes com vários tipos de câncer avançado durante a radioterapia, ${ }^{13}$ (A) e também melhora a qualidade de vida em pacientes com câncer de mama durante o tratamento oncológico (quimioterapia, radioterapia ou cirurgia $)^{14}$ (A).

Um treino supervisionado de flexibilidade melhora de forma significativa a qualidade de vida de pacientes com câncer, de jovem a média idade, pouco tempo após a quimioterapia ${ }^{15}(\mathbf{A})$.

\section{RECOMENDAÇÃo}

Embora os poucos estudos com pacientes com câncer de pulmão não tenham revelado resultados consistentes ${ }^{4-7}$ recomendamos para estes pacientes a prática regular (pelos menos 150 minutos por semana, distribuídos ao longo de cinco dias) e, inicialmente, supervisionada de exercícios físicos, aeróbicos e resistidos, isolados ou associados, pois os poucos estudos com essa população não são suficientes e adequadamente desenhados para concluirmos que o exercício não traz benefício nos sintomas da doença, na fadiga e na qualidade de vida nessa população, nossa recomendação baseia-se em benefícios do exercício físico comprovados em estudos cuja população foi pacientes com diversos tipos de câncer e em diversas fases do tratamento oncológico.

\section{O EXERCÍCIO FÍSICO PRÉ-OPERATÓRIO DIMINUI AS COMPLICAÇÕES PULMONARES NO PÓS-OPERATÓRIO EM PACIENTES COM CÂNCER DE PULMÃo?}

A ocorrência de complicações pós-operatórias diminui significativamente de $16,7 \%$ para $3 \%$ quando é realizada fisioterapia respiratória e exercícios aeróbios de forma intensiva na semana que precede a cirúrgia de ressecção tumoral de pulmão quando comparado à fisioterapia respiratória regular $(p<0,05) \cdot{ }^{16}$

Fisioterapia pré-operatória e reabilitação pulmonar trazem benefícios para pacientes com indicação de ressecção pulmonar por neoplasia. Observa-se melhora da capacidade e preservação da função pulmonar após a cirurgia, porém não há associação clara na redução da incidência de complicações pós-operatórias. ${ }^{17}$

\section{RECOMENDAÇÃo}

Podem-se recomendar exercícios aeróbios associados à fisioterapia respiratória de forma intensiva no pré-operatório de pacientes hígidos com indicação de ressecção pulmonar por neoplasia de pulmão, pois demonstram ter capacidade de melhorar a capacidade pulmonar e, eventualmente, minimizar o risco de complicações pós-operatórias. 


\section{O EXERCÍCIO FíSICO NO PÓS-OPERATÓRIO IMEDIATO DIMINUI A} DISPNEIA EM PACIENTES COM CÂNCER DE PULMÃO?

Um programa de exercício físico aeróbio personalizado e supervisionado, consistindo em três sessões em cicloergômetro em dias alternados por 14 semanas, praticado a $60 \%$ da frequência cardíaca máxima por 15 a 20 minutos no início, com aumentos de duração de 5 minutos a cada semana de acordo com a tolerância do paciente (avaliada por sinais hemodinâmicos, saturação de $\mathrm{O}_{2}$ e aumento na frequência cardíaca máxima até $70 \%$ ) em pacientes recém-submetidos à lobectomia ou pneumectomia por câncer de pulmão, é capaz de reduzir a queixa de dispneia (médias e desvios-padrão pré e pós-intervenção: $17 \pm 5$ e $20 \pm 5 ; p=0,007$ ) medida na avaliação de bem-estar funcional da escala de qualidade de vida FACT-L (Functional Assesment Of Cancer Therapy-Lung). ${ }^{18}$

Um programa personalizado de caminhada, instituído para pacientes com câncer de pulmão sem indicação cirúrgica ou para pacientes no pós-operatório, com intensidade e frequência dos treinos de caminhada aumentando de acordo com a monitorização de queixa de dispneia, com objetivo de completar um percurso de 10 metros, o maior número de vezes possível, até sintoma de pré-exaustão, mensurado pelas Escala de Borg, duas vezes por semana por sete semanas, melhora a queixa de dispneia medida pela escala de Qualidade de Vida EORTC QLQ-C30 (na média: nota 50 para 33), porém não demonstra superioridade sobre grupo controle no pós-operatório imediato e nem entre os grupos cirúrgico e não-cirúrgico, mas sugere melhora qualitativa no bem estar geral associado à percepção do impacto da dispneia na capacidade física e na redução do ciclo-vicioso da dispneia-sedentarismo. Os pacientes que evitaram exercício por medo da dispneia aderiram à proposta terapêutica com redução dos sintomas, ainda que com poder fraco para conclusões estatísticas ${ }^{7}$ (B).

\section{Recomendação}

Pode ser recomendado exercício aeróbio supervisionado com intensidade moderada no pós-operatório imediato de pacientes submetidos à ressecção pulmonar por neoplasia de pulmão, especialmente sem indicação de quimioterapia adjuvante posterior. A intervenção é segura e promove melhora qualitativa da queixa de desconforto respiratório global ao exercício e da dispneia

\section{O EXERCíCIO FÍSICO NO PÓS-OPERATÓRIO IMEDIATO DIMINUI A FADIGA EM PACIENTES COM CÂNCER DE PULMÃO?}

Um programa de exercício físico aeróbio personalizado e supervisionado, consistindo em três sessões em cicloergômetro em dias alternados por 14 semanas, praticado a $60 \%$ da frequência cardíaca máxima por 15 a 20 minutos no início, com aumentos de duração de 5 minutos a cada semana de acordo com a tolerância do paciente (avaliada por sinais hemodinâmicos, saturação de $\mathrm{O}_{2}$ e aumento na frequência cardíaca máxima até $70 \%$ ) em pacientes recém-submetidos à lobectomia ou pneumectomia por câncer de pulmão, é capaz de melhorar o cansaço e desconforto nas pernas medido pela avaliação de fadiga (médias e desvios-padrão pré e pósintervenção: $19 \pm 8$ e $12 \pm 8 p=0,03$ ) na escala de qualidade de vida FACT-L (Functional Assesment Of Cancer Therapy-Lung). ${ }^{18}$

\section{ReCOMENDAÇÃo}

Pode ser recomendado exercício aeróbio supervisionado com intensidade moderada no pós-operatório imediato de pacientes submetidos à ressecção pulmonar por neoplasia de pulmão, especialmente sem indicação de quimioterapia adjuvante posterior. A intervenção é segura e promove melhora qualitativa da queixa de fadiga ao exercício.

\section{UM PROGRAMA PSICOEDUCATIVO COM TERAPIA COGNITIVO-COMPORTAMENTAL MELHORA A QUALIDADE DE VIDA EM PACIENTES COM CÂNCER DE PULMÃO?}

Um programa psicoeducativo, baseado em habilitação e adoção, por parte dos pacientes, de comportamentos adaptativos para atrasar o agravamento dos sintomas, com os seguintes componentes: informação preparatória, discussão da experiência do sintoma, exploração dos significados e manifestações associadas aos sintomas, consultoria em estratégias de enfrentamento, e treinamento e prática de relaxamento muscular progressivo, realizado para pacientes com câncer de pulmão avançado (estadio 3 e 4) em radioterapia paliativa, é capaz de melhorar os sintomas de ansiedade $(p=0,001)$, fadiga $(p=0,011)$, falta de ar $(p=0,002)$, habilidade funcional $(p=0,001)$ e sintoma "cluster" $(p=0,003)^{19}(\mathbf{A})$.

Uma intervenção psicoeducacional, uma vez por semana, durante quatro semanas, realizada por enfermeiras, abordando orientações educacionais, alimentares e aconselhamento antes do final da vida e posterior acompanhamento mensal até a morte, melhora a qualidade de vida $(p=0,02)$ e o humor $(p=0,03)$, mas não melhora a intensidade dos sintomas $(p=0,24)$, nem reduz os dias de internação hospitalar, ou em unidade de terapia intensiva, nem a procura pelos serviços de emergência, em comparação com pacientes recebendo cuidados oncológicos habituais ${ }^{20}(\mathbf{A})$.

A terapia cognitivo-comportamental aplicada por enfermeira, em quatro sessões (inicial, após 10, 20 e 32 semanas), em pacientes com câncer de mama (38\%), pulmão (35\%) e outros tumores sólidos (cólon, ginecológico, linfoma e útero), podem melhorar a funcionalidade, mas o efeito na melhora da funcionalidade é menor quanto maior os sintomas depressivos apresentados pelos pacientes $(p<0,01)^{21}$ (B).

\section{RECOMENDAÇÃo}

Recomendamos um programa psicoeducativo, realizado por profissional de saúde, preferencialmente psicólogo capacitado e com experiência no cuidado de pacientes com doenças crônicas, voltado para orientações para comportamentos adaptativos no dia-a-dia, busca de estratégias de enfrentamento e uso de técnicas de relaxamento para pacientes com câncer de pulmão, em qualquer fase do tratamento e da doença, porém um programa mais intenso para pacientes com estadios mais avançados e durante tratamento específico.

\section{UM PROGRAMA DE CONDICIONAMENTO FÍSICO EM PACIENTES} COM CÂNCER DE PULMÃO MELHORA A TOLERÂNCIA AO EXERCÍCIO?

Um programa supervisionado interdisciplinar de reabilitação, de no máximo 20 sessões, pelo menos cinco vezes por semana, composto de atividade aeróbica em cicloergômetro durante pelo menos 30 minutos e de intensidade moderada ( $60 \%$ a $80 \%$ da carga máxima), aumenta a tolerância ao exercício físico no teste de caminhada de 6 minutos com média de aumento de 95,6 metros em relação a um grupo controle sem intervenção $(p<0,001)^{22}$ (A). O mesmo tipo de treino de atividade física em cicloergômetro e esteira, realizado diariamente, por pelo menos 20 minutos cada atividade, por oito semanas, e com intensidade variando de 60 a $80 \%$ da carga máxima e valores de 4 a 6 na escala de Borg, além de fisioterapia motora para 
manutenção da amplitude de movimento e atividade anaeróbica de fortalecimento de 3 a 15 repetições com carga entre $30-60 \%$ de uma repetição máxima (1-RM), leva à melhora do desempenho no teste de caminhada de 6 minutos: pacientes conseguem caminhar uma distância 43,2\% maior $(p=0,002)^{23}(\mathbf{B})$.

Um programa de exercícios supervisionados no pós-operatório imediato, duas vezes ao dia, e mantido como atividade domiciliar, também diária duas vezes ao dia, supervisionada ao menos uma vez ao mês durante 12 semanas, com atividades motoras para a manutenção da amplitude de movimento, treino de força, marcha, atividade livre em bicicleta estacionária, durante 5 a 10 minutos, cada modalidade, e de intensidade moderada variando de 60 a $80 \%$ da frequência cardíaca máxima, não melhora a tolerância ao exercicio no teste de caminhada de 6 minutos $(p=0.47)^{4}$ (A).

\section{ReCOMENDAÇÃo}

Programas supervisionados interdisciplinares com treinos aeróbicos de intensidade moderada (60 a 80\% da frequência cardíaca máxima) por pelo menos 30 minutos por sessão, três vezes por semana, podem ser recomendados, pois há evidências de que melhoram a tolerância ao exercício, mesmo que a atividade física seja realizada em cicloergômetro ou bicicleta estacionária.

\section{A SUPLEMENTAÇÃO DE ERGOGÊNICOS (B-HIDROXI-B-METILLBUTI- RATO (HMB), CREATINA) ASSOCIADA AO EXERCÍCIO FÍSICO REDUZ A FADIGA EM PACIENTES COM CÂNCER DE PULMÃO?}

Suplementação de creatina, com dose inicial de $5 \mathrm{~g} \mathrm{4x/dia,} \mathrm{du-}$ rante uma semana, seguindo de $2,5 \mathrm{~g} 2 \mathrm{x} / \mathrm{dia}$, até completar oito semanas, melhora a bioimpedância e a relação massa extracelular/ massa celular corporal $(p=0,043)$. Tal efeito é maior em pacientes sem doença metastática e que receberam apenas fluoracil/acido fólico como quimioterapia. Pacientes com tumores em estágio avançado ou recebendo quimioterapia mais agressiva contendo oxaplatina ou irinocetan não têm benefício com a suplementação da creatina ${ }^{24}(\mathbf{A})$.

Pacientes com caquexia por diversos tipos de tumores sólidos avançados (Estágio III e V) ou com doença metastática, com perda ponderal entre 2 e $10 \%$, não melhoram o peso após suplementação com composto nutricional de L-arginina (14 g/dia), glutamina (14 g/dia) e betahidroxi betametilbutirato ( $3 \mathrm{~g} / \mathrm{dia})$, durante 8semanas de suplementação $(p=0,08)^{25}$ (B).

Pacientes com diversos tipos de tumores sólidos avançados (Estágio IV), com perda ponderal $>5 \%$ e em curso de diferentes tratamentos, não melhoram o peso por aumento de massa magra, após suplementação com composto nutricional de L-arginina (14 g/dia), glutamina (14 g/dia) e betahidroxi betametilbutirato ( $3 \mathrm{~g} / \mathrm{dia})$, durante 24semanas $(p>0,25)^{26}$ (B).

\section{RECOMENDAÇÃo}

Não é possível recomendar o uso destes suplementos para meIhora da fadiga em pacientes com câncer de pulmão por falta de evidências na literatura para essa população específica, e com desfecho de fadiga. Entretanto, conforme exposto acima, há evidências de melhora de alguns parâmetros de bioimpedância em pacientes com neoplasia colorretal e a melhora desses parâmetros tem se mostrado associada à melhor sobrevida e preditores de prognóstico nessa população e isso deve ser levado em consideração na decisão para uso destes suplementos. Mais estudos são necessário parasse avaliar o efeito do uso de ergogênicos como a creatina e B- hidroxi-B- metilbutirato (HMB) para melhora da fadiga em pacientes com câncer de pulmão.

\section{O GUARANÁ ("PAULLINIA CUPANA") MELHORA A FADIGA EM PA- CIENTES COM CÂNCER DE PULMÃO?}

O extrato do guaraná (Paullinia cupana) na dose de $50 \mathrm{mg}$, duas vezes ao dia, por um período de 21 dias, pode melhorar a fadiga em pacientes com câncer de mama durante quimioterapia em comparação com placebo $(p<0,01)$, não produz eventos adversos ou toxicidades maiores, não piora a qualidade do sono e também não causa ansiedade ou depressão nessa população ${ }^{27}$ (B).

\section{RECOMENDAÇÃo}

O extrato do guaraná na dose de $50 \mathrm{mg}$, duas vezes ao dia, pode melhorar a fadiga em pacientes com câncer de pulmão, embora o estudo que demonstrou benefício tenha sido realizado em pacientes com câncer de mama. Porém, devemos fazer uso com cautela, principalmente em pacientes com cardiopatia, hipertensão arterial sistêmica e idosos, até que mais estudos mostrem a dose segura em relação a efeitos adversos, como aumento da pressão arterial e da frequência cardíaca.

\section{REFERÊNCIAS}

1. Ferlay J, Shin HR, Bray F, Forman D, Mathers C, Parkin DM. Estimates of worldwide burden of cancer in 2008: GLOBOCAN 2008. Int J Cancer. 2010;127(12):2893-917. DOI: http://dx.doi.org/10.1002/ijc.25516

2. Jemal A, Bray F, Center MM, Ferlay J, Ward E, Forman D. Global cancer statistics. CA Cancer J Clin. 2011;61(2):69-90. DOI: http://dx.doi.org/10.3322/caac.20107

3. Chute CG, Greenberg ER, Baron J, Korson R, Baker J, Yates J. Presenting conditions of 1539 population-based lung cancer patients by cell type and stage in New Hampshire and Vermont. Cancer. 1985;56(8):2107-11.

4. Arbane G, Tropman D, Jackson D, Garrod R. Evaluation of an early exercise intervention after thoracotomy for non-small cell lung cancer (NSCLC), effects on quality of life, muscle strength and exercise tolerance: randomised controlled trial. Lung Cancer. 2011;71(2):229-34 DOI: http://dx.doi.org/10.1016/j. lungcan.2010.04.025

5. Wall LM. Changes in hope and power in lung cancer patients who exercise. Nurs Sci Q. 2000;13(3):234-42. DOI: http://dx.doi.org/10.1177/08943180022107627

6. Bobbio A, Chetta A, Ampollini L, Primomo GL, Internullo E, Carbognani $P$, et al. Preoperative pulmonary rehabilitation in patients undergoing lung resection for non-small cell lung cancer. Eur J Cardiothorac Surg. 2008;33(1):95-8. DOI: http:// dx.doi.org/10.1016/j.ejcts.2007.10.003

7. Andersen $\mathrm{AH}$, Vinther A, Poulsen LL, Mellemgaard A. Do patients with lung cancer benefit from physical exercise? Acta Oncol. 2011;50(2):307-13. DOI: http://dx.doi. org/10.3109/0284186X.2010.529461

8. Van Weert E, May AM, Korstjens I, Post WJ, van der Schans $C P$, van den Borne B, et al. Cancer-related fatigue and rehabilitation: a randomized controlled multicenter trial comparing physical training combined with cognitive-behavioral therapy with physical training only and with no intervention. Phys Ther. 2010;90(10):1413-25. DOI: http://dx.doi.org/10.2522/ptj.20090212

9. Adamsen L, Quist M, Andersen C, Møller T, Herrstedt J, Kronborg D, et al. Effect of a multimodal high intensity exercise intervention in cancer patients undergoing chemotherapy: randomised controlled trial. BMJ. 2009;339:b3410.

10. Segal RJ, Reid RD, Courneya KS, Sigal RJ, Kenny GP, Prud'Homme DG, et al. Randomized controlled trial of resistance or aerobic exercise in men receiving radiation therapy for prostate cancer. J Clin Oncol. 2009;27(3):344-51. DOI: http:// dx.doi.org/10.1200/JCO.2007.15.4963

11. Dimeo FC, Thomas F, Raabe-Menssen C, Pröpper F, Mathias M. Effect of aerobic exercise and relaxation training on fatigue and physical performance of cancer patients after surgery. A randomised controlled trial. Support Care Cancer. 2004;12(11):774-9. DOI: http://dx.doi.org/10.1007/s00520-004-0676-4 
12. Courneya KS, Mackey JR, Bell GJ, Jones LW, Field CJ, Fairey AS. Randomized controlled trial of exercise training in postmenopausal breast cancer survivors: cardiopulmonary and quality of life outcomes. J Clin Oncol. 2003;21(9):1660-8. DOI: http://dx.doi.org/10.1200/JCO.2003.04.093

13. Cheville AL, Girardi J, Clark MM, Rummans TA, PittelkowT, Brown P, et al. Therapeutic exercise during outpatient radiation therapy for advanced cancer: Feasibility and impact on physical well-being. Am J Phys Med Rehabil. 2010;89(8):611-9. PMID: 20531162 DOI: http://dx.doi.org/10.1097/PHM.0b013e3181d3e782

14. Haines TP, Sinnamon P, Wetzig NG, Lehman M, Walpole E, Pratt T, et al. Multimodal exercise improves quality of life of women being treated for breast cancer, but at what cost? Randomized trial with economic evaluation. Breast Cancer Res Treat. 2010;124(1):163-75. DOI: http://dx.doi.org/10.1007/s10549-010-1126-2

15. Thorsen L, Skovlund E, Strømme SB, Hornslien K, Dahl AA, Fosså SD. Effectiveness of physical activity on cardiorespiratory fitness and health-related quality of life in young and middle-aged cancer patients shortly after chemotherapy. J Clin Oncol. 2005;23(10):2378-88. DOI: http://dx.doi.org/10.1200/JCO.2005.04.106

16. Pehlivan E, Turna A, Gurses A, Gurses HN. The effects of preoperative short-term intense physical therapy in lung cancer patients: a randomized controlled trial. Ann Thorac Cardiovasc Surg. 2011;17(5):461-8. DOI: http://dx.doi.org/10.5761/ atcs.oa.11.01663

17. Nagarajan K, Bennett A, Agostini P, Naidu B. Is preoperative physiotherapy/ pulmonary rehabilitation beneficial in lung resection patients? Interact Cardiovasc Thorac Surg. 2011;13(3):300-2. DOI: http://dx.doi.org/10.1510/icvts.2010.264507

18. Jones LW, Eves ND, Peterson BL, Garst J, Crawford J, West MJ, et al. Safety and feasibility of aerobic training on cardiopulmonary function and quality of life in postsurgical nonsmall cell lung cancer patients: a pilot study. Cancer. 2008;113(12):3430-9. DOI: http://dx.doi.org/10.1002/cncr.23967

19. Chan CW, Richardson A, Richardson J. Managing symptoms in patients with advanced lung cancer during radiotherapy: results of a psychoeducational randomized controlled trial. J Pain Symptom Manage. 2011;41(2):347-57. DOI: http://dx.doi.org/10.1016/j.jpainsymman.2010.04.024
20. Bakitas M, Lyons KD, Hegel MT, Balan S, Brokaw FC, Seville J, et al. Effects of a palliative care intervention on clinical outcomes in patients with advanced cancer: the Project ENABLE II randomized controlled trial. JAMA. 2009;302(7):741-9. DOI: http://dx.doi.org/10.1001/jama.2009.1198

21. Doorenbos A, Given B, Given C, Verbitsky N. Physical functioning: effect of behavioral intervention for symptoms among individuals with cancer. Nurs Res. 2006;55(3):161-71. DOI: http://dx.doi.org/10.1097/00006199-200605000-00002

22. Cesario A, Ferri L, Galetta D, Pasqua F, Bonassi S, Clini E, et al. Post-operative respiratory rehabilitation after lung resection for non-small cell lung cancer. Lung Cancer. 2007;57(2):175-80. DOI: http://dx.doi.org/10.1016/j.lungcan.2007.02.017

23. Spruit MA, Janssen PP, Willemsen SC, Hochstenbag MM, Wouters EF. Exercise capacity before and after an 8-week multidisciplinary inpatient rehabilitation program in lung cancer patients: a pilot study. Lung Cancer. 2006;52(2):257-60. DOI: http://dx.doi.org/10.1016/j.lungcan.2006.01.003

24. Norman K, Stübler D, Baier P, Schütz T, Ocran K, Holm E, et al. Effects of creatine supplementation on nutritional status, muscle function and quality of life in patients with colorectal cancer-a double blind randomised controlled trial. Clin Nutr. 2006;25(4):596-605. DOI: http://dx.doi.org/10.1016/j.clnu.2006.01.014

25. Berk L, James J, Schwartz A, Hug E, Mahadevan A, Samuels M, et al. A randomized, double-blind, placebo-controlled trial of a beta-hydroxyl beta-methyl butyrate, glutamine, and arginine mixture for the treatment of cancer cachexia (RTOG 0122). Support Care Cancer. 2008;16(10):1179-88. DOI: http://dx.doi.org/10.1007/ s00520-008-0403-7

26. May PE, Barber A, D'Olimpio JT, Hourihane A, Abumrad NN. Reversal of cancerrelated wasting using oral supplementation with a combination of beta-hydroxybeta-methylbutyrate, arginine, and glutamine. Am J Surg. 2002;183(4):471-9. PMID: 11975938 DOI: http://dx.doi.org/10.1016/S0002-9610(02)00823-1

27. Oliveira Campos MP, Riechelmann R, Martins LC, Hassan BJ, Casa FB, Del Giglio A. Guarana (Paullinia cupana) improves fatigue in breast cancer patients undergoing systemic chemotherapy. J Altern Complement Med. 2011;17(6):505-12. 